$\begin{array}{ll}\text { Research Square } & \text { Preprints are preliminary reports that have not undergone peer review. } \\ \text { They should not be considered conclusive, used to inform clinical practice, } \\ \text { or referenced by the media as validated information. }\end{array}$

\title{
Correlation between Red Blood Cell Distribution width, Neutrophil to Lymphocyte Ratio and Neutrophil to Platelet Ratio with 3-month Prognosis of Patients with Intracerebral Hemorrhage: A Retrospective Study
}

\author{
Sheida Shaafi \\ Tabriz University of Medical Sciences \\ Ehsan Bonakdari \\ Tabriz University of Medical Sciences \\ Yalda Sadeghpour \\ Tabriz University of Medical Sciences \\ Seyed Aria Nejadghaderi ( $\square$ ariang20@gmail.com) \\ Shahid Beheshti University of Medical Sciences
}

\section{Research Article}

Keywords: Red cell distribution width, Intracerebral hemorrhage, Neutrophil to lymphocyte ratio, Neutrophil to platelet ratio, Prognosis

Posted Date: January 4th, 2022

DOl: https://doi.org/10.21203/rs.3.rs-1200296/v1

License: @ (1) This work is licensed under a Creative Commons Attribution 4.0 International License. Read Full License 


\section{Abstract}

Background: Red cell distribution width (RDW) is a parameter that indicates the heterogeneity of red blood cell size and could be as a prognostic factor in some diseases. Also, intracerebral hemorrhage $(\mathrm{ICH})$ is considered a vascular event with a high mortality rate. Here, we aimed to examine the role of RDW, neutrophil to lymphocyte (NLR) and neutrophil to platelet ratios (NPR) in predicting the prognosis of patients with $\mathrm{ICH}$.

Methods: This is a retrospective cohort study which conducted on 140 patients with ICH admitted to the neurology ward and intensive care unit (ICU) in Imam Reza Hospital, Tabriz, Iran. Demographic data, National Institutes of Health Stroke Scale (NIHSS), and complete blood count (CBC) test parameters evaluated within 24 hours after hospitalization. These variables collected and re-evaluated three months later.

Results: The mean age of the study population was $61.14( \pm 16)$ years and $51 \%$ were male. RDW had a significant positive correlation with hemoglobin concentration $(r=0.3 ; p<0.001)$ and significant negative correlation with $\mathrm{MCHC}(r=-0.57 ; p<0.001)$ and neutrophil count $(r=-0.235$; $p=0.006)$. Also, the mean NLR $(p=0.05)$, neutrophil and platelet count $(p=0.05)$, NIHSS $(p=0.05)$, and RDW $(p=0.01)$ had a significant difference between the deceased patients and those who partially recovered after 3 months. Moreover, the results of logistic regression showed variables including RDW ( $p=0.006)$ and NIHSS $(p<0.001)$ levels were correlated significantly with mortality.

Conclusion: RDW could be an appropriate prognostic factor and predictor in determining 3-months survival in ICH. Nevertheless, further largescale prospective cohorts might be needed to evaluate the associations.

\section{Introduction}

Red cell distribution width (RDW) is a parameter that represent the dispersion of erythrocyte volume. The coefficient of size changes of red blood cells is more accurate than structure of red blood cells in peripheral blood smears $(1,2)$. RDW values can be determined easily using automatic flow cytometer as part of a standard complete blood count (3). Many studies have shown that abnormal RDW values are highly associated with mortality and poor prognosis (4-6) in various diseases such as acute coronary syndrome, cerebral ischemic disease, heart failure and peripheral vascular disease (7-9). Higher RDW values impairs blood circulation and oxygen supply, so elevated RDW values are associated with more severe and unfavorable outcome (10).

Neutrophil to lymphocyte ratio (NLR) and neutrophil to platelet ratio (NPR) are indices which can represent the activities of the inflammatory complex and inflammatory response in the vascular bed (11). NLR is a useful biomarker for evaluation inflammatory response following intracerebral hemorrhage $(\mathrm{ICH})$, and it is associated with prognosis, in which the more severe the inflammatory response, the more aggravated nerve injury is expected $(12,13)$.

In 2019, the total prevalent cases of ICH were 20.66 million (95\% uncertainty interval (UI): 18.02-23.42), which showed a $58.0 \%$ increase since 1990. Furthermore, ICH, with an increase of $25.0 \%$ since 1990, was accounted for 68.57 million (95\% UI: 63.27-73.68) disability-adjusted lifeyears in 2019 (14). Also, it is associated with various complications such as hydrocephalus and cerebral edema which could be even lifethreatening (15). Therefore, early diagnosis and prediction of its prognosis could be helpful for the management in clinical settings.

The predictive value of some blood markers in patients with different types of strokes have been evaluated in previous research (16). However, there is a lack of the correlation between different complete blood count (CBC) parameters simultaneously with prognosis of ICH. Therefore, the aim of this study is to describe the 3-month prognostic utility of RDW, NLR, and NPR in patients with ICH.

\section{Material And Methods}

\section{Study design and subject population}

The target population in the present retrospective cohort study was patients with ICH who were referred to Imam Reza Hospital, Tabriz, Iran from 2014 to 2019. Using random sampling method, a total number of 140 participants were enrolled into the study. Patients over 18 years old and a definitive diagnosis of first-time cerebral hemorrhage with complete laboratory information were included in the study. Exclusion criteria were age under 18 years, history of any type of cancer, end-stage liver disease, acute or chronic renal failure, receiving blood products in previous 3 months of hospitalization, $\mathrm{ICH}$ following trauma, and long-term duration (i.e. above 48 hours) between ICH and hospitalization.

Written informed consent was obtained from all of the participants at the beginning of the study. The study protocol was approved by the ethics committee of Tabriz University of Medical Sciences (Ethics Code: IR.TBZMED.REC.1398.797). All methods were performed in accordance with the national guidelines and regulations. 


\section{Data collection}

Patient information including age, gender, underlying diseases (e.g. hypertension, diabetes, hyperlipidemia and ischemic heart disease), CBC parameters including white blood cell count, lymphocyte and neutrophil count, hemoglobin concentration, hematocrit, erythrocyte count, mean corpuscular hemoglobin concentration (MCHC), RDW and platelet count, neurological function and severity of the stroke measured using the National Institutes of Health Stroke Scale (NIHSS) score were collected. Moreover, mortality at discharge, within 28-day, and 3-month later were collected.

\section{Outcomes}

The primary outcome was to determine the relationship between RDW, NLR and NPR with the 3-month prognosis of participants. The secondary outcomes were to determine the RDW, NLR and NPR mean values in patients with ICH. Also, we aimed to evaluate the short-term mortality rate and the effects of using RDW, NLR and NPR simultaneously in prediction of ICH prognosis.

\section{Data analysis}

Collected data were analyzed by SPSS statistical analysis software version 18 (SPSS Inc., Chicago, IL, USA). First of all, Smirnov-Kolmogorov test was used to assess the normal distribution of variables. Demographic variables were analyzed by descriptive statistical methods and presented with percentage, frequency and mean \pm standard deviation (SD). Analysis of quantitative variables was performed using independent sample T-test or Mann-Whitney U test. Moreover, long-term predictive value analysis was performed by Cox regression model. The p-values less than 0.05 was considered as statistically significant. Logistic regression was also used to investigate the effect of variables on 3 month mortality. In this test, the dependent variable was 3-month outcomes (i.e. death/recovery) and independent variables were RDW, platelet count, NIHSS, MCHC, RBC, NPR and NLR.

\section{Results}

\section{Baseline characteristics}

The average age of 140 patients was 61.14 years with the SD of 16 and ranged from 18 to 96 years old. There were 71 males and 69 females in our sample (Table 1). Hypertension and hyperlipidemia were the most and the least common underlying diseases among the participants (Table 2). The median (interquartile range [IQR]) of hematoma volume in alive and deceased groups were 10 (5-25) and 35 (13-60) milliliter, respectively. Moreover, there were 113 supratentorial hematomas, including putamen/internal capsule $(n=47)$, lobar hematoma $(n=11)$, thalamus $(n=24)$, and intraventricular $(n=31)$. In addition, infratentorial hematomas were located in pons $(n=15)$, midbrain $(n=1)$, and cerebellum $(n=11)$. 
Table 1

Descriptive statistics of numerical variables.

\begin{tabular}{|lllll|}
\hline & Minimum & Maximum & Mean & Standard deviation \\
\hline Age(year) & 18 & 96 & 61.14 & 16.05 \\
\hline WBC(× 1000) & 4.7 & 32.5 & 11.63 & 5.26 \\
\hline Lymphocyte $(\times$ 1000) & 2.5 & 52.20 & 15.70 & 10.44 \\
\hline Neutrophil(× 1000) & 7.20 & 95.80 & 77.80 & 14.92 \\
\hline Hemoglobin (g/dl) & 5.70 & 35.20 & 13.79 & 2.71 \\
\hline Hematocrit $(\%)$ & 19.70 & 57.40 & 42.17 & 5.54 \\
\hline RBC(× 1000) & 2.4 & 6.80 & 4.72 & 0.677 \\
\hline MCHC (g/dl) & 16.60 & 36 & 32.09 & 1.93 \\
\hline RDW $(\%)$ & 11.40 & 26.6 & 14.02 & 2.06 \\
\hline Platelet(x 1000) & 103 & 458 & 232.860 & 66.80 \\
\hline NIHSS & 0 & 40 & 15.86 & 15.09 \\
\hline NLR & 0.78 & 36.44 & 8.25 & 6.29 \\
\hline NPR & 0.008 & 0.78 & 0.05 & 0.09 \\
\hline
\end{tabular}

Table 2

Descriptive statistics of categorical variables.

\begin{tabular}{|lllll|}
\hline Underlying disease & \multicolumn{4}{c}{ Number $(\%)$} \\
\cline { 2 - 5 } & & Both sexes $(\mathrm{n}=140)$ & Males $(\mathrm{n}=71)$ & Females $(\mathrm{n}=69)$ \\
\hline Diabetes & Yes & $23(16.4)$ & $9(12.7)$ & $14(20.3)$ \\
\cline { 2 - 5 } & No & $117(83.6)$ & $62(87.3)$ & $55(79.7)$ \\
\hline Hyperlipidemia & Yes & $7(5.0)$ & $3(4.2)$ & $4(5.8)$ \\
\cline { 2 - 5 } & No & $133(95.0)$ & $68(95.8)$ & $65(94.2)$ \\
\hline Hypertension & Yes & $106(75.7)$ & $48(67.6)$ & $58(84.0)$ \\
\cline { 2 - 5 } Coronary artery disease & No & $34(24.3)$ & $23(32.4)$ & $11(16.0)$ \\
\cline { 2 - 5 } & Yes & $25(17.9)$ & $15(21.1)$ & $10(14.5)$ \\
\cline { 2 - 5 } & No & $115(82.1)$ & $56(78.9)$ & $59(85.5)$ \\
\cline { 2 - 5 } & & & & \\
\hline
\end{tabular}

\section{Outcomes}

In this study, the mortality of patients in a short period of 28-day hospitalization and in a period of 3 months was considered. Results of mortality study in two time periods showed that in the final result of 3 months, 11 people were added to the number of patients who died in comparison with 28-day study period and the deceased participants increased from $33.6 \%$ to $41.4 \%$ (Figure 1 ).

\section{Correlates of RDW}

RDW was significantly correlated with hemoglobin concentration $(r=0.30 ; p<0.001)$ positively and with neutrophil count $(r=-0.23 ; p=0.006)$ and MCHC ( $r=-0.57 ; p<0.001)$ in a negative way (Table 3).

Table 3

Results of RDW correlation test and other research variables. 


\begin{tabular}{|c|c|c|c|c|c|c|c|c|c|c|c|c|}
\hline & & NIHSS & Platelet & $\mathrm{MCHC}$ & $\mathrm{RBC}$ & Hematocrit & Hemoglobin & Neutrophil & Lymphocyte & WBC & NPR & NLR \\
\hline \multirow[t]{2}{*}{ RDW } & $r$ & $0.007-$ & -0.114 & -0.568 & 0.202 & -0.21 & 0.300 & -0.235 & -0.70 & -0.19 & 0.035 & 0.007 \\
\hline & $\mathrm{P}$ & 0.936 & 0.190 & 0.000 & 0.21 & 0.811 & 0.000 & 0.006 & 0.423 & 0.829 & 0.68 & 0.94 \\
\hline
\end{tabular}

Abbreviations: WBC: white blood cell; RBC: red blood cell; MCHC: mean corpuscular hemoglobin concentration; RDW: red cell distribution width; NIHSS: National Institutes of Health Stroke Scale; NLR: neutrophil to lymphocyte ratio; NPR: neutrophil to platelet ratio.

\section{Predictors of 3-month prognosis of patients with ICH}

To investigate the effect of the variables on the mortality of patients, the mean of the variables by patients' final outcome (death or recovery) was assessed and compared with T-test between the two groups. Higher NIHSS scores were statistically significant with death within 3 months $(p<0.01)$, while higher neutrophil count was associated with higher survival $(p=0.04)$ (Table 4 and Figure S1).

Table 4

Comparison of the mean of research variables by 3-month prognosis of patients

\begin{tabular}{|c|c|c|c|c|c|c|}
\hline Variables & 3-month prognosis & Number & Mean & Standard deviation & $P(t$-test) & Abbreviations: WBC: white blood cell; RBC: red \\
\hline \multirow[t]{2}{*}{ NLR } & Dead & 57 & 9.43 & 7.10 & \multirow[t]{2}{*}{0.05} & hemoglobin concentration; RDW: red cell \\
\hline & Alive & 83 & 7.44 & 5.56 & & distribution width; NIHSS: National Institutes of \\
\hline \multirow[t]{2}{*}{ NPR } & Dead & 57 & 0.06 & 0.087 & \multirow[t]{2}{*}{0.41} & Health Stroke Scale; NLR: neutrophil to \\
\hline & Alive & 83 & 0.05 & 0.093 & & ratio. \\
\hline \multirow[t]{2}{*}{ RDW } & Dead & 54 & 14.37 & 2.18 & \multirow[t]{2}{*}{0.10} & \\
\hline & Alive & 80 & 13.78 & 1.95 & & Independent variables \\
\hline \multirow[t]{2}{*}{ NIHSS } & Dead & 57 & 27.56 & 14.23 & \multirow[t]{2}{*}{0.00} & predicting mortality of \\
\hline & Alive & 83 & 7.83 & 9.33 & & patients with ICH \\
\hline \multirow[t]{2}{*}{ Platelet } & Dead & 57 & 219.64 & 57.79 & \multirow[t]{2}{*}{0.05} & Regression analysis showed that independent \\
\hline & Alive & 83 & 241.93 & 71.27 & & variables, including RDW ( $p=0.006)$, platelet \\
\hline \multirow[t]{2}{*}{$\mathrm{MCHC}$} & Dead & 57 & 32.14 & 1.50 & \multirow[t]{2}{*}{0.80} & $\begin{array}{l}\text { count }(p=0.047) \text {, and NIHSS }(p<0.001) \text { with } \\
\text { negative coefficients significantly impact on 3- }\end{array}$ \\
\hline & Alive & 83 & 32.05 & 2.18 & & month mortality, which means higher values of \\
\hline \multirow[t]{2}{*}{ RBC } & Dead & 56 & 4.60 & 0.82 & \multirow[t]{2}{*}{0.07} & the variables are associated with more deaths. \\
\hline & Alive & 81 & 4.81 & 0.54 & & $\begin{array}{l}\mathrm{RBC} \text { count }(p<0.001) \text { With positive coefficlents } \\
\text { was effective on the mortality variable }\end{array}$ \\
\hline \multirow[t]{2}{*}{ WBC } & Dead & 57 & 12.42 & 5.13 & \multirow[t]{2}{*}{0.13} & significantly so that increasing of the two \\
\hline & Alive & 83 & 11.08 & 5.30 & & variables leads to less deaths (Table 5). \\
\hline \multirow[t]{2}{*}{ Lymphocyte } & Dead & 57 & 13.85 & 9.98 & \multirow[t]{2}{*}{0.08} & Table 5 \\
\hline & Alive & 83 & 16.97 & 10.62 & & Logistic regression results in predicting the \\
\hline \multirow[t]{2}{*}{ Neutrophil } & Dead & 57 & 80.70 & 11.85 & \multirow[t]{2}{*}{0.04} & final mortality of patients by independent \\
\hline & Alive & 83 & 75.81 & 16.47 & & varlables. \\
\hline \multirow[t]{2}{*}{ Hemoglobin } & Dead & 57 & 13.48 & 2.45 & \multirow[t]{2}{*}{0.25} & Abbreviations: SE: standard error; RBC: red \\
\hline & Alive & 83 & 14.01 & 2.87 & & hemoglobin concentration; RDW: red cell \\
\hline \multirow[t]{2}{*}{ Hematocrit } & Dead & 57 & 41.78 & 6.96 & \multirow[t]{2}{*}{0.5} & distribution width; NIHSS: National Institutes of \\
\hline & Alive & 83 & 42.45 & 4.33 & & $\begin{array}{l}\text { Health Stroke Scale; NLR: neutrophil to } \\
\text { lymphocyte ratio; NPR: neutrophil to platelet }\end{array}$ \\
\hline
\end{tabular}

ratio. 


\begin{tabular}{|c|c|c|c|c|c|c|}
\hline Independent variables & Beta coefficient & SE & Wald & $\mathrm{p}$ & $\operatorname{Exp}(B)$ & Receiver operating characteristic (ROC) curve was \\
\hline RDW & -0.542 & 0.192 & 7.45 & 0.006 & 0.592 & 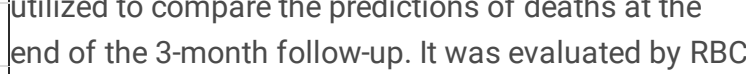 \\
\hline NLR & -0.62 & 0.043 & 2.065 & 0.151 & 0.940 & NIHSS, NPR, NLR, RDW variables. We found that the \\
\hline NPR & -0.906 & 2.44 & 0.137 & 0.711 & 0.404 & NPR variable had the highest area below the curve, \\
\hline RBC & 1.365 & 0.510 & 7.164 & 0.007 & 3.917 & patient's death prediction (Figure 2 and Table S1). \\
\hline $\mathrm{MCHC}$ & -0.326 & 0.230 & 2.005 & 0.157 & 0.722 & \\
\hline Platelet & -0.10 & 0.005 & 3.943 & 0.047 & 1.010 & Discussion \\
\hline NIHSS & -0.138 & 0.023 & 34.959 & 0.00 & 0.871 & The purpose of this study was to determine the \\
\hline Constant & 12.330 & 8.570 & 2.070 & 0.150 & 226340.54 & $\begin{array}{l}\text { predictive value of RDW, NLR, and NPR in the 3-month } \\
\text { mortality of patients with intracerebral hemorrhage. }\end{array}$ \\
\hline
\end{tabular}

The findings of the present study showed that hemoglobin has a significant positive correlation with RDW, while MCHC and neutrophil count has a negative one. Moreover, RDW, RBC, platelet count, and NIHSS scores are independent predictors of 3-month mortality in patients with $\mathrm{ICH}$.

RDW as an available laboratory factor, which is a marker of the variation in volume of red blood cells (3), can be used as prognostic factor in various health conditions (17). Moreover, it is associated with chronic inflammation and oxidative stress (5). Thus, it is reasonable that high RDW values can be known as an integrated risk factor for sub-optimal general health condition which is accompanied by a reduction in correction, improvement and systemic defense capability $(5,9)$. In our study the role of RDW factor in the prognosis and mortality of stroke patients with ICH in a period for 3 months was assessed and was shown that RDW value is significantly higher in deceased patients in comparison to alive patients with ICH. The results of the study conducted by Lorente et al. among 117 patients with spontaneous ICH showed that RDW during the first week was higher in deceased than surviving patients ( $\leq \leq 0.001)$, which is in accordance to our findings (18). Furthermore, the article by Kaya et al. conducted on 153 participants concluded that RDW is an important hematopoiesis indicator for occurrence of stroke in patients with heart failure which reveals that high RDW is associated with the development of stroke and is strongly associated with cardiovascular causes (10). A study by Moreno et al. also showed that RDW is a strong indicator for stroke (19). Moreover, another study by Kara et al. concluded that RDW, an easily accessible and inexpensive test, is potentially an important parameter in diagnosing stroke and may predict prognosis. Also, in this study also NIHSS was used to determine the severity of stroke and it was found that the increase in stroke severity is associated with higher RDW and RDW is a strong predictor of stroke severity (20).

Of note, a wide range of diseases and even physiological conditions lead to changes in the level of RDW in the human body. For example, different types of anemia inflammatory diseases and cancers can cause changes in the size of red blood cells (21). Thus, RDW may not be specific enough to screen for stroke in many patients. One of the most appropriate scales to assess the severity of stroke is NIHSS which measures the severity of a neurological deficit. The NIHSS is a common diagnostic tool for quickly assessing the severity of a stroke and related neurologic deficits which is used in determining the prognosis. NIHSS had the highest association with mortality rate in stroke patients, so this variable was significantly higher among the deceased group. Also, the area under the ROC curve for this variable shows the meaningful power of predicting the death of patients by this variable (22). In addition to RDW and NIHSS, NLR, platelets and neutrophils were significantly effective in determining the prognosis of the intracerebral hemorrhage (23).

The $N L R$ is simply calculated as a ratio by division the number of neutrophils to the number of lymphocytes in peripheral blood and measures the inflammatory response (24). Acute inflammatory response occurs in some serious conditions such as ischemic stroke, cerebral hemorrhage, and acute coronary syndrome. Therefore, this marker can be used to determine the prognosis and evaluation of disease outcome in the mentioned diseases $(11,24,25)$. In this regard, our results also showed that higher RDW, NIHSS, RBC, and platelet count increases the risk of short-term and long-term mortality in patients with stroke.

Numerous studies have shown that high levels of neutrophils are sensitive markers for determining mortality (23). Jickling et al. and Maestrini et al. showed that high neutrophil level is associated with high infarct volume and poor prognosis $(26,27)$. In addition to its function in the homeostasis, platelets also play a role in the inflammatory responses. In fact, platelets can facilitate neutrophil entry into the brain parenchyma and higher neutrophil counts will lead to the more severe damage (28).

We acknowledge that our study had several limitations. First, since it was a retrospective study, restrictions in data collection, participant inclusion, and lack of a control group exist. Second, it was conducted in a single hospital. Third, the variables were only measured in two time points in 28-days and 3-months and they did not be remeasured during the period of hospitalization. Fourth, the follow-up was limited to 3 months and no ling-term follow-up was conducted. 


\section{Conclusion}

The current study demonstrated that increased levels of RDW, NLR, neutrophil and platelet are associated with the severity of ICH which leads to an increased mortality rate in these patients. According to the results of the present study, it is suggested to increase the sample size in future studies to increase the accuracy of the results, control and delete other probable variables affecting RDW in the sample, including of the information about the region and volume of hemorrhage and evaluate the relationship between study variables and the prognosis of patients in different age groups.

\section{Declarations}

\section{Acknowledgments}

We are grateful to all who participated in this study.

\section{Declaration of Conflicting Interests}

The authors declared no potential conflict of interest.

\section{Funding}

The authors received no financial support for the research.

\section{Availability of data and material}

The datasets generated and/or analyzed during the current study are not publicly available due for they are personal data but are available from the corresponding author on reasonable request.

\section{Contributions}

S.S. and Y.S. conceptualized and designed the study; E.B. implemented the study and collected the data; E.B., Y.S., and S.A.N. wrote the first draft of the paper and critically revised the manuscript. All the authors reviewed and approved the final draft of the manuscript.

\section{Ethics approval and consent to participate}

Written informed consent was obtained from all of the participants at the beginning of the study. The study protocol was approved by the ethics committee of Tabriz University of Medical Sciences (Ethics Code: IR.TBZMED.REC.1398.797). All methods were performed in accordance with the national guidelines and regulations.

\section{Consent for publication}

Written informed consent was obtained from all of the participants at the beginning of the study. All methods were performed in accordance with the national guidelines and regulations.

\section{References}

1. Lippi G, Plebani M. Red blood cell distribution width (RDW) and human pathology. One size fits all. Clinical Chemistry and Laboratory Medicine (CCLM). 2014;52(9):1247-9.

2. Şenol K, Saylam B, Kocaay F, Tez M. Red cell distribution width as a predictor of mortality in acute pancreatitis. Am J Emerg Med. 2013;31(4):687-9.

3. Sadaka F, O'Brien J, Prakash S. Red Cell Distribution Width and Outcome in Patients With Septic Shock. Journal of Intensive Care Medicine. 2012;28(5):307-13.

4. Feng G-H, Li H-P, Li Q-L, Fu Y, Huang R-B. Red blood cell distribution width and ischaemic stroke. Stroke Vasc Neurol. 2017;2(3):172-5. 
5. Förhécz Z, Gombos T, Borgulya G, Pozsonyi Z, Prohászka Z, Jánoskuti L. Red cell distribution width in heart failure: Prediction of clinical events and relationship with markers of ineffective erythropoiesis, inflammation, renal function, and nutritional state. American Heart Journal. 2009;158(4):659-66.

6. Sousa R, Gonçalves C, Guerra IC, Costa E, Fernandes A, do Bom Sucesso M, et al. Increased red cell distribution width in Fanconi anemia: a novel marker of stress erythropoiesis. Orphanet Journal of Rare Diseases. 2016;11(1):102.

7. Adamsson Eryd S, Borné Y, Melander O, Persson M, Smith JG, Hedblad B, et al. Red blood cell distribution width is associated with incidence of atrial fibrillation. J Intern Med. 2014;275(1):84-92.

8. Danese E, Lippi G, Montagnana M. Red blood cell distribution width and cardiovascular diseases. J Thorac Dis. 2015;7(10):E402-E11.

9. Gul M, Uyarel H, Ergelen M, Karacimen D, Ugur M, Turer A, et al. The relationship between red blood cell distribution width and the clinical outcomes in non-ST elevation myocardial infarction and unstable angina pectoris: A 3-year follow-up. Coronary artery disease. 2012;23:330-6.

10. Kaya A, Isik T, Kaya Y, Enginyurt O, Gunaydin ZY, Iscanli MD, et al. Relationship Between Red Cell Distribution Width and Stroke in Patients With Stable Chronic Heart Failure: A Propensity Score Matching Analysis. Clinical and Applied Thrombosis/Hemostasis. 2013;21(2):1605.

11. Tamhane UU, Aneja S, Montgomery D, Rogers E-K, Eagle KA, Gurm HS. Association Between Admission Neutrophil to Lymphocyte Ratio and Outcomes in Patients With Acute Coronary Syndrome. The American Journal of Cardiology. 2008;102(6):653-7.

12. Cai L, Zeng H, Tan X, Wu X, Qian C, Chen G. The Role of the Blood Neutrophil-to-Lymphocyte Ratio in Aneurysmal Subarachnoid Hemorrhage. Front Neurol. 2021;12:671098-.

13. Xiao L, Zheng H, Li J, Wang Q, Sun H. Neuroinflammation Mediated by NLRP3 Inflammasome After Intracerebral Hemorrhage and Potential Therapeutic Targets. Molecular Neurobiology. 2020;57(12):5130-49.

14. Feigin VL, Stark BA, Johnson CO, Roth GA, Bisignano C, Abady GG, et al. Global, regional, and national burden of stroke and its risk factors, 1990-2019: a systematic analysis for the Global Burden of Disease Study 2019. The Lancet Neurology. 2021.

15. Park C, Charalambous LT, Yang Z, Adil SM, Hodges SE, Lee H-J, et al. Inpatient mortality and healthcare resource utilization of nontraumatic intracerebral hemorrhage complications in the US. Journal of Neurosurgery JNS. 2021:1-10.

16. Ören $\mathrm{O}$, Haki C, Kaya H, Yüksel M. Predictive value of admission neutrophil/lymphocyte ratio in symptomatic intracranial hemorrhage after stroke thrombolysis. Neurological Sciences. 2021.

17. Ani C, Ovbiagele B. Elevated red blood cell distribution width predicts mortality in persons with known stroke. J Neurol Sci. 2009;277(12):103-8.

18. Lorente L, Martín MM, González-Rivero AF, Pérez-Cejas A, Sabatel R, Ramos L, et al. Red blood cell distribution width and mortality of spontaneous intracerebral hemorrhage patients. Clinical Neurology and Neurosurgery. 2020;195:106066.

19. Ramírez-Moreno JM, Gonzalez-Gomez M, Ollero-Ortiz A, Roa-Montero AM, Gómez-Baquero MJ, Constantino-Silva AB. Relation between Red Blood Cell Distribution Width and Ischemic Stroke: A Case-Control Study. International Journal of Stroke. 2013;8(6):E36-E.

20. Kara H, Degirmenci S, Bayir A, Ak A, Akinci M, Dogru A, et al. Red cell distribution width and neurological scoring systems in acute stroke patients. Neuropsychiatr Dis Treat. 2015;11:733-9.

21. Silva Litao MK, Kamat D. Back to Basics: Red Blood Cell Distribution Width: Clinical Use beyond Hematology. Pediatrics in Review. 2018;39(4):204.

22. Gattringer T, Posekany A, Niederkorn K, Knoflach M, Poltrum B, Mutzenbach S, et al. Predicting Early Mortality of Acute Ischemic Stroke. Stroke. 2019;50(2):349-56.

23. Yang W, Liu Y. Platelet-lymphocyte ratio is a predictor of venous thromboembolism in cancer patients. Thromb Res. 2015;136(2):212-5.

24. Ozcan Cetin EH, Cetin MS, Aras D, Topaloglu S, Temizhan A, Kisacik HL, et al. Platelet to Lymphocyte Ratio as a Prognostic Marker of InHospital and Long-Term Major Adverse Cardiovascular Events in ST-Segment Elevation Myocardial Infarction. Angiology. 2015;67(4):336-45.

25. Kundi H, Balun A, Cicekcioglu H, Cetin M, Kiziltunc E, Cetin ZG, et al. The relation between platelet-to-lymphocyte ratio and Pulmonary Embolism Severity Index in acute pulmonary embolism. Heart \& Lung. 2015;44(4):340-3.

26. Jickling GC, Liu D, Ander BP, Stamova B, Zhan X, Sharp FR. Targeting Neutrophils in Ischemic Stroke: Translational Insights from Experimental Studies. Journal of Cerebral Blood Flow \& Metabolism. 2015;35(6):888-901.

27. Maestrini I, Strbian D, Gautier S, Haapaniemi E, Moulin S, Sairanen T, et al. Abstract W P51: A Higher Neutrophil Count Before Thrombolysis Is Associated With Worse Outcome. Stroke. 2015;46(suppl_1):AWP51-AWP.

28. He W, Ruan Y, Yuan C, Cheng Q, Cheng H, Zeng Y, et al. High Neutrophil-to-Platelet Ratio Is Associated With Hemorrhagic Transformation in Patients With Acute Ischemic Stroke. Front Neurol. 2019;10(1310). 
Figures

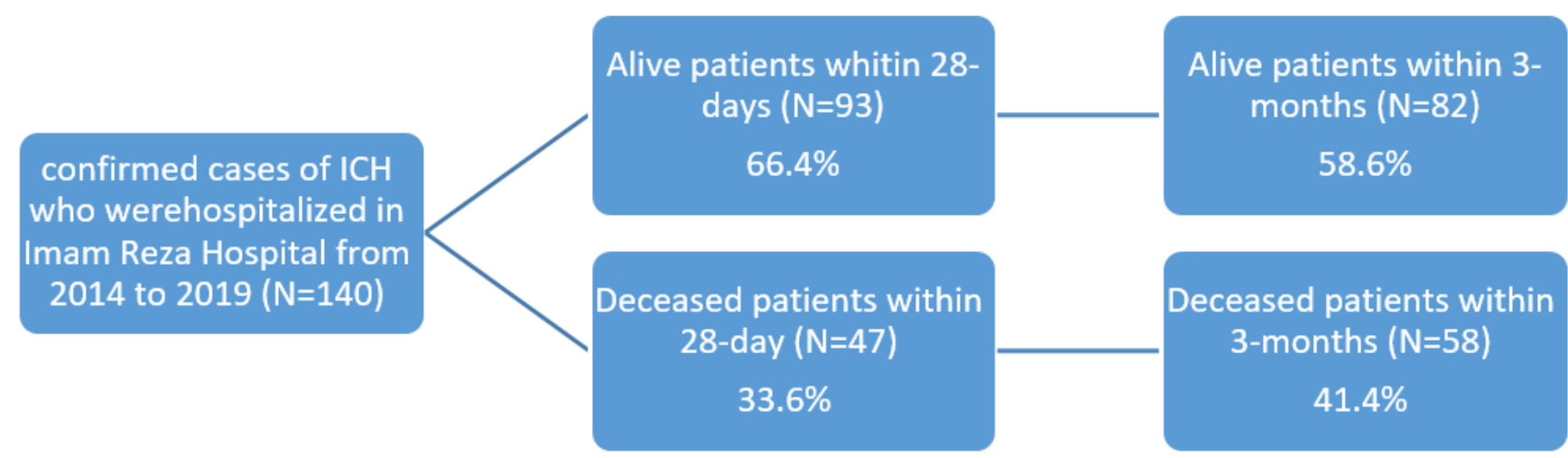

Figure 1

28-day and 3-month outcomes of the study.

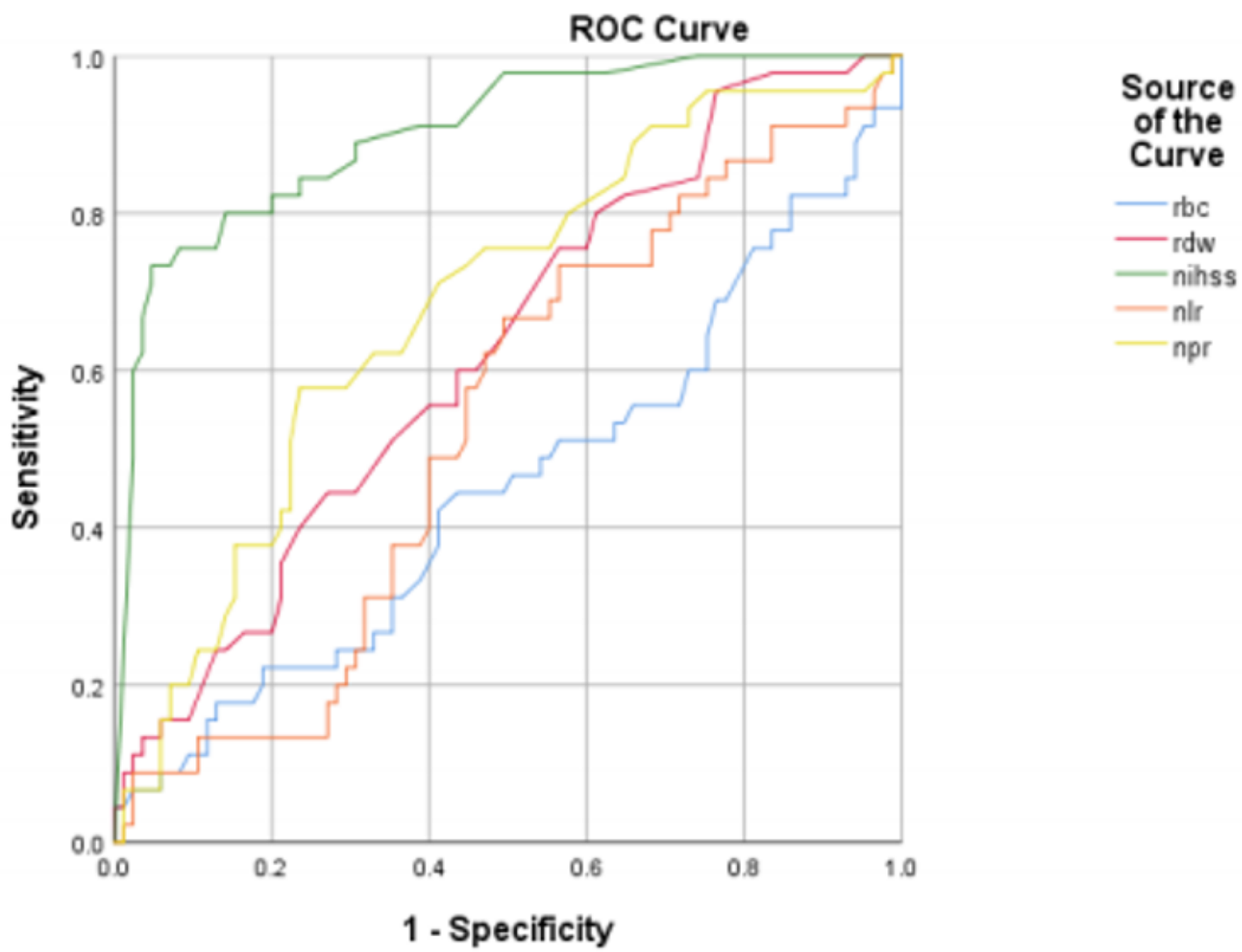

Figure 2

ROC curve of research variables to predict the death of patients at the end of the 3-month period.

Abbreviations: RBC: red blood cell; RDW: red cell distribution width; NIHSS: National Institutes of Health Stroke Scale; NLR: neutrophil to lymphocyte ratio; NPR: neutrophil to platelet ratio. 


\section{Supplementary Files}

This is a list of supplementary files associated with this preprint. Click to download.

- Supplementarymaterial.docx 